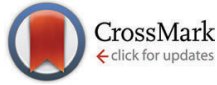

Cite this: Phys. Chem. Chem. Phys., 2015, 17, 1077

Received 6th September 2014, Accepted 13th November 2014 DOI: $10.1039 / c 4 c p 04008 b$

www.rsc.org/pccp

\section{Whirl-enhanced continuous wave laser trapping of particlest:}

\author{
S. Bartkiewicz and A. Miniewicz*
}

\begin{abstract}
Tightly focused laser beams can trap micro- and nanoparticles suspended in liquids in their focal spots enabling different functionalities including 3D manipulations and assembling. Here, we report on remarkably strong liquid-liquid phase separation and crystallization experiments in para-nitroaniline dissolved in 1,4-dioxane. For optical trapping of para-nitroaniline we used low-power, weakly focused light beam from continuous-wave laser partially absorbed by the solute. The experiments were performed in solution deposited on glass with an upper free-surface and solution contained between two glass plates. The usual gradient force field and scattering force solely are insufficient to properly describe the observed particle gathering effects extending far beyond the optical trap potential. The concept of whirl-enhanced and temperature assisted optical trapping is postulated. The relative simplicity of the used geometry for trapping will broaden the understanding of the light-matter interaction and promises the widespread application of the observed effect in optically controlled crystallization.
\end{abstract}

\section{A Introduction}

Laser trapping is a phenomenon enabling gathering by focused laser beam macromolecules, micelles, nanoparticles, and molecular clusters in solution at room temperature. It is commonly accepted that laser trapping of particles can be interpreted by geometrical optics and Maxwell-Boltzmann electromagnetic theory. ${ }^{1-3}$ The particles that can effectively be trapped by focused laser beam must have dimensions ranging from several nanometers to micrometers. The reason for that is that the optical trapping potential energy exerted on nanometer-size objects in solution at room temperature must be higher or at least comparable to their thermal energy resulting from Brownian motion and collision interactions. Therefore objects much smaller in size than microparticles or several tens of nanometer nanoparticles, such as individual molecules, cannot be trapped by light. Light can also transfer a torque force to an ensemble of trapped particles, e.g. by transfer of angular orbital momentum carried by a doughnut-shaped Laguerre-Gauss beam to the particles. $^{4-7}$ Sometimes beam that does not carry angular

Institute of Physical and Theoretical Chemistry, Department of Chemistry, Wroclaw University of Technology, Wybrzeze Wyspianskiego 27, 50-370 Wroclaw, Poland.E-mail: andrzej.miniewicz@pwr.edu.pl

$\dagger$ This work is dedicated to Dr Francois Kajzar (France) on the occasion of his 70th birthday.

\# Electronic supplementary information (ESI) available: Four short video movies described in the text as SIM1 to SIM4 accompany this work and illustrate the dynamics of droplet formation and whirl behavior and the table with the relevant physicochemical properties of the solvent and solute. See DOI: 10.1039/ c4cp04008b orbital momentum can induce coherent rotation of the particles around the beam propagation direction if the trapped particles themselves can support a suitable momentum. ${ }^{8}$ This can happen in the specific alignment of particles with respect to the beam axis and the beam polarization. ${ }^{9}$ Laser trapping and manipulation has already found numerous applications in science and technology. ${ }^{10-19}$ By using various spatial laser beam intensity distributions and different multi-focus and holographic beam geometries laser-tweezers proved to be a quite versatile optical tool for studying microfluidics, optically driven micromachines, microrheology and flow field measurements. ${ }^{7,20-23}$

The optical trapping of very large molecules has been already demonstrated, namely for polymers, ${ }^{24,25} \mathrm{~J}$-aggregates, ${ }^{26}$ aminoacid clusters, ${ }^{27}$ micelles, ${ }^{28}$ quantum dots ${ }^{29}$ and nanoparticles. ${ }^{30-33}$ However, the trapping of non-aggregating molecules has not been demonstrated so far. In a recent paper written by Yuyama et al. ${ }^{34}$ a millimeter-scale dense liquid droplet formation of glycine in $\mathrm{D}_{2} \mathrm{O}$ due to focused laser beam was described and explained on the basis of photon pressure exerted on glycine clusters. The laser light intensity used in this experiment was $\sim 0.4 \mathrm{GW} \mathrm{cm} \mathrm{cm}^{-2}$, so intense light is able to trap objects of size larger than $17 \mathrm{~nm}$.

A $56 \mathrm{MW} \mathrm{cm}^{-2}$ infrared laser light intensity has been used to induce optical reorientation and creation of metastable domains in the nematic liquid crystal of $4^{\prime}$-pentyl-4cyanobiphenyl. ${ }^{35}$ Nanoplasmonics has extended the field of optical trapping down to the nanometer scale with exciting possibilities for studying single proteins and nanoparticles. This technique benefits from the strong perturbation of the nanoparticle due to the local electromagnetic environment in the metallic nanoaperture. In this case trapping is approximately 
$3000 \times$ more efficient than the conventional one and particles of $12 \mathrm{~nm}$ in size and even single proteins have been trapped with double-nanohole (DNH) structures with 1-10 mW power. ${ }^{36}$

However, as stated by Ashkin ${ }^{2}$ interesting applications of optical manipulation techniques exist in other diverse areas of physics and chemistry, especially in the field of microchemistry where polymerization, ablation, crystal growth and other microfabrication techniques were demonstrated. ${ }^{37-41}$ In this work we report on microdroplet formation and microcrystal growth under cw laser light illumination in a new solute-solvent system as compared to that reported by Yuyama et al. ${ }^{34}$ However, to observe the qualitatively the same phenomenon we used light intensity roughly six orders of magnitude lower than that used in the quoted work. ${ }^{34}$ Therefore the explanation of this laser-assisted molecular assembling is of primary importance as one has to answer what kind of enhancement in lightmatter interaction is taking place. At the trapping wavelength of $405 \mathrm{~nm}$, one can expect an increase in the temperature of the solution along the beam path. Thermal expansion of the fluid may result in convective flow ${ }^{42}$ extending over a distance that is large compared to the size of laser beam.

\section{B Results and discussion}

\section{B1 Laser assisted liquid-liquid phase separation}

Laser trapping that we have observed was realized for the first time in the system of the $p$-nitroaniline compound ( $p$-NA, $\mathrm{C}_{6} \mathrm{H}_{6} \mathrm{~N}_{2} \mathrm{O}_{2}$ ), the well-known NLO chromophore, which was dissolved in 1,4-dioxane $\left(\mathrm{C}_{4} \mathrm{H}_{8} \mathrm{O}_{2}\right)$, a common organic solvent. The details of the considered phenomenon and the physicochemical properties of the solute and solvent are given in ESI. $\neq$ A few other systems, such as solute-solvent, that we have investigated showed similar effects. To observe the optical trapping we placed the $p$-nitroaniline microcrystals on the microscopic glass slide and then by adding a few drops of 1,4-dioxane solvent we traced their complete dissolution under the optical microscope equipped with a CCD camera. The solution was transparent and its color was hardly visible. The upper surface of the droplet was free and was in contact with air as schematically shown in Fig. 1 illustrating the geometry of the experiment.

We estimated the initial liquid layer thickness to amount to $h \sim 200-400 \mu \mathrm{m}$. Illumination of the droplet was performed using a linearly polarized Gaussian beam from a laser diode source loosely focused on the target and incident from the bottom through a glass plate. The beam parameters are as follows: wavelength $\lambda=405 \mathrm{~nm}$, on sample spot diameter $2 w_{0}=$ $200 \mu \mathrm{m}$, the depth of focus equals twice the Rayleigh length $2 z_{0}$ amounted to $155 \mathrm{~mm}$ and total power $P$ in the range 10 to $100 \mathrm{~mW}$ which resulted in the maximum value of light intensity at the focus $I_{0}=64-640 \mathrm{~W} \mathrm{~cm}^{-2}$. Laser light intensity is related to average electric field strength $\left\langle E_{0}\right\rangle$ through equation $I_{0}=1 / 2\left(\varepsilon_{0} n_{\mathrm{d}} c E_{0}^{2}\right)\left(\mathrm{W} \mathrm{m}^{-2}\right)$. For 1,4-dioxane solvent having refractive index $n_{\mathrm{d}}=1.404$ calculations yield the value of $\left\langle E_{0}\right\rangle=18.5-58.6 \mathrm{kV} \mathrm{m}^{-1}$, respectively. Immediately after launching the laser light a circular-shaped yellowish colored area appeared
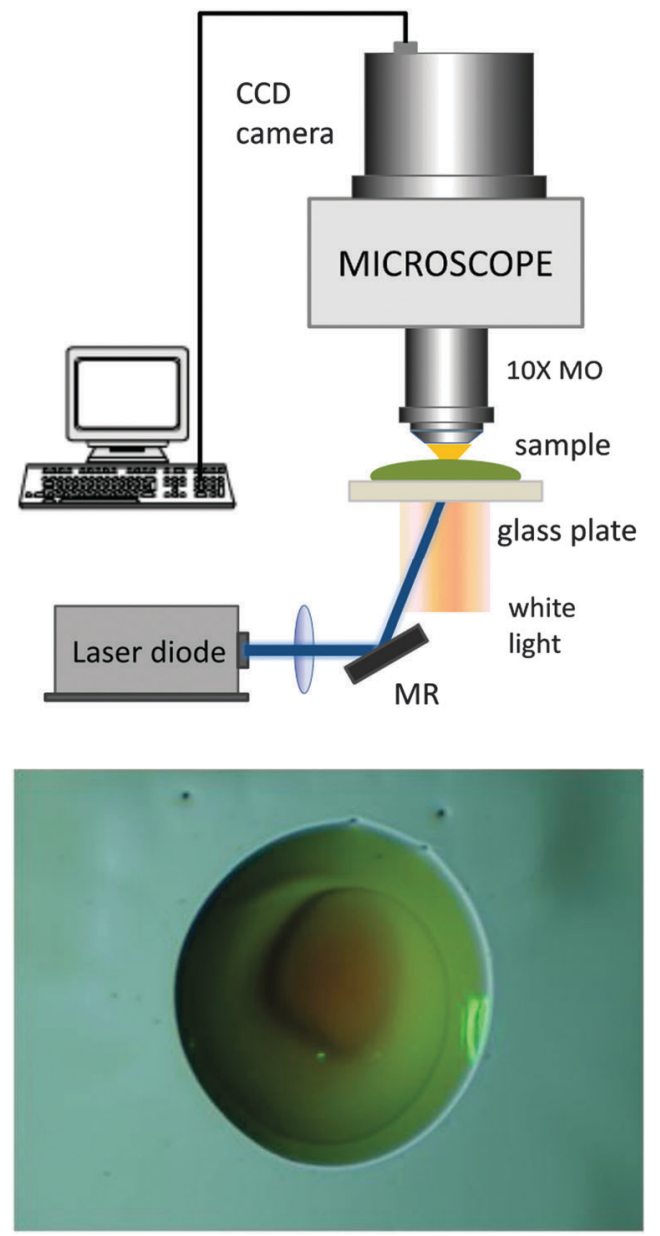

Fig. 1 Scheme of the experimental setup and the photograph of the droplet of 1,4-dioxane with high content of $p$-nitroaniline formed due to $\mathrm{cW}$ laser light beam illumination of wavelength $405 \mathrm{~nm}$ and peak intensity $I_{0} \sim 500 \mathrm{~W} \mathrm{~cm}^{-2}$. The diameter of the droplet shown in the photograph is about $0.5 \mathrm{~mm}$.

at the laser spot position. The initially small convex-shaped droplet around the focal spot steeply grew in size then stabilized within seconds reaching a diameter of $0.5-1 \mathrm{~mm}$, i.e. much wider than the beam diameter ( $c f$. Fig. 2c).

The more dense color in the droplet center suggested the higher concentration of $p$-NA in the center and/or thickness of the layer at the central droplet place. The frequently observed spiral line suggested the slow azimuthal rotation of the liquid. The droplet is uniquely stabilized by the presence of the laser beam as a few seconds after switching-off the light the droplet disappears, i.e. $p$-NA molecules are dispersed by diffusing to the surrounding solution. Subsequent light opening results exactly in the same phenomenon, i.e. the reappearance of the colored nucleus followed by its growth. Astonishingly, after a while the border of the circle sharpens and is very well established. In fact a droplet of dense liquid containing $p$-NA is formed in the remaining 1,4-dioxane solution depleted of $p$-NA after a relatively short time of illumination. The almost saturated $p$-nitroaniline liquid condensate shows its own surface tension and can be deformed easily or even left apart by sudden 


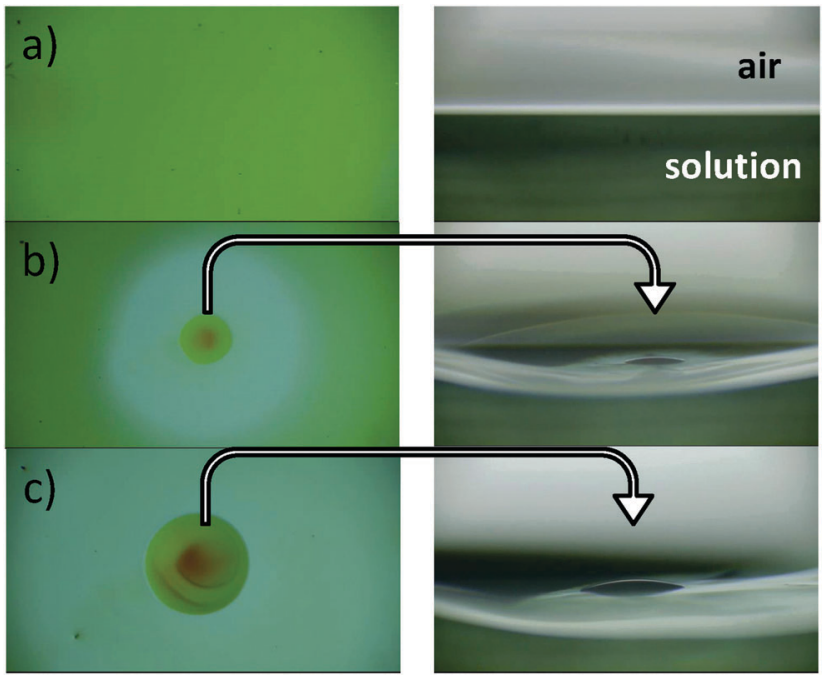

Fig. 2 Temporal evolution of formation of a dense droplet of $p$-nitroaniline in 1,4-dioxane upon cw laser irradiation. Normal to the surface (left side) and lateral (right side) photographs as a function of illumination time illustrate the trapping process. (a) Flat surface prior illumination; (b) 2 seconds after illumination onset one observes the accumulation of colored $p$-NA solution at the beam center accompanied by layer thickness decrease around the illuminated area seen as a white circle; (c) 10 seconds after illumination onset - the droplet is larger than the beam diameter and the area around it forms a thin layer that extends far from the central point.

movement of the beam. The droplet is evidently trapped by the laser beam as it moves relative to the smooth lateral movements of a laser spot. A similar phenomenon to that described here was named the laser trapping-induced liquid-liquid phase separation $^{34,43}$ and abbreviated as LLPS. In ESI $\neq$ a short video film SIM1 illustrates how a rapid movement of the laser spot outside the droplet leaves it alone and a small new one is formed in the place of the beam center. The previously formed droplet starts to behave chaotically and disappears in a diffusive manner within seconds. Frequently, one can observe, how the newly created trapping center sucks the colored (saturated) solution left after the previous trapping center and this results in a much faster growth in the size of the new droplet. Interestingly $p$-NA is attracted from distances much larger than the light spot size excluding the action of light dragging forces alone but involving light-induced fluid flows centered toward the focal point.

Due to the choice of colorless solvent and colored solute the difference between the circular droplet condensate and the surrounding solution is high and well visible in white light under an optical microscope in transmission mode ( $c f$. Fig. 2). The whiter circular shadow surrounding the nucleus ( $c f$. Fig. 2b) and appearing in a short time interval after launching light is a result of formation of a thin layer of solution around the droplet. This was evidenced by us using the observation of droplet formation from the direction parallel to the glass plate surface ( $c f$. right side of Fig. 2). The thin layer formation around it does not prevent the growth of droplets, contrary to this it is able to feed the central droplet with the $p$-nitroaniline particles until the laser light is present. The nature of the solution flow toward the beam spot remains open.

\section{B2 Physics of laser assisted droplet formation}

Observing the details of light-induced droplet formation we conclude that we deal in this case with the peculiar "optical trapping" mechanism that is strongly enhanced by coupling of the light-induced electrostrictive effect with hydrodynamic heat and mass transport (convection). At a $405 \mathrm{~nm}$ wavelength there is a non-negligible light absorption of $p$-NA, as the first absorption band of $p$-NA in 1,4-dioxane is centered at $352 \mathrm{~nm}$ at $c=$ $1.9 \times 10^{-5} \mathrm{M}$. Even initially weak light absorption present in the solution will lead to temperature increase along the beam axis inducing a vertical temperature gradient. The time of the onset of temperature non-uniformity is $d^{2} / \kappa \approx 4.4 \mathrm{~ms}$ (where beam diameter $d=0.2 \mathrm{~mm}$ and $\kappa \approx 9 \times 10^{-6} \mathrm{~m}^{2} \mathrm{~s}^{-1}$ is the thermal diffusivity of 1,4-dioxane). Associated with it is the onset of convective mass and heat flow toward the free surface where the heat exchange is the fastest. The increased temperature at the free surface will reduce the surface tension. This could be seen as a slight surface depression formed initially on the top of the layer.

We observed it ( $c f$. Fig. 2b) by placing the microscope objective at the angle of $90^{\circ}$ to the surface normal. Immediately after at the bottom of this area emerges a new droplet of dense $p$-NA in 1,4-dioxane with its distinct phase boundary. This droplet enlarges at the cost of the available $p$-NA in the surrounding solution ( $c f$. Fig. 2c). At the same time the layer of solution around the growing droplet becomes thinner finally reaching the thickness of a few microns. This does not stop the collection of $p$-NA molecules from outside, they flow to the beam center enlarging the droplet. The whirl sucks the cooler liquid from the bottom, heat it and transport it upward the surface where the cooling occurs, partially also due to intense solvent evaporation. The $p$-NA enriched condensate surrounded by solution forms a kind of light-induced convex lens. In separate experiments we observed how this lens magnifies the microscopic size objects when they enter the area of the droplet. The droplet during its formation can be illuminated with $532 \mathrm{~nm}$ laser light and is able to reflect the incoming laser light giving nice interference patterns in the far field on the screen.

In order to help the reader imagine the phenomenon shown in Fig. 2 we sketched it schematically in Fig. 3a. The beam width, liquid layer thickness and droplet size and shape (enriched in the $p$-NA liquid-like condensate showing its own surface) all maintain proportions observed under a microscope. The above described experiment of laser induced molecular optical trapping is very clear and repeatable but its understanding to date is far from being satisfactory due to the complexity and interplay between all involved phenomena.

\section{B3 Whirls induced by absorbed laser light}

In this report we propose a new concept of the laser trapping mechanism that can be quite universal in its nature regarding "optical trapping” of small polarizable molecules. We think that in the observed process a distinct role is played by the hydrodynamic whirl formation that enhances the transport of $p$-NA molecules toward the laser beam center which itself has 
a)
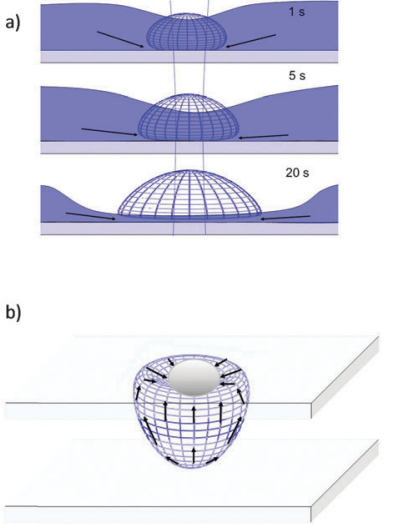

c)

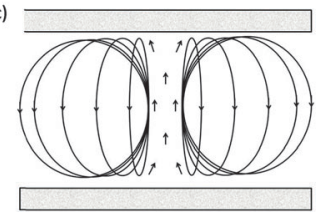

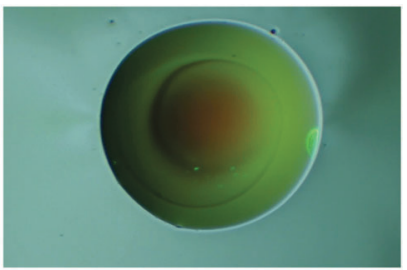
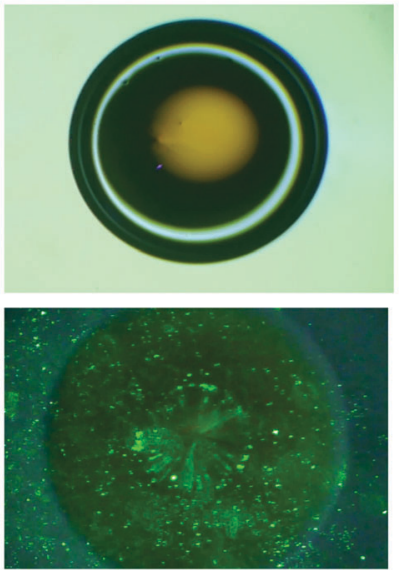

Fig. 3 Illustration of various types of laser induced whirls (side views) and their photographs (top views). (a) Scheme of light-induced liquid-liquid phase separation. Deformation of the solution free surface is accompanied by the formation of $p$-NA enriched droplets. Laser beam is directed vertically and reaches the glass from the bottom, its width is illustrated by vertical lines, and arrows indicate the flow of liquid towards the beam center where $p$-NA is accumulated. (b) Scheme of a whirl formed in the liquid layer confined between two glass plates under laser beam illumination and stabilized by a trapped air bubble at its top. Arrows indicate the movement of mass of liquid inside the whirl. (c) Scheme of whirl with small luminescent particles vigorously rotating along the circular loop trajectories.

the ability to attract polarizable particles. The formation of whirl (movement of liquid in the form of closed loops as shown schematically in Fig. $3 \mathrm{~b}$ and c) explains why under certain conditions the trapped matter (here $p$-NA) finally extends in lateral size much wider than the laser beam diameter itself. Similar, but also qualitatively different, effects of laser 'trapping' can be seen when the upper surface of the solution layer is covered by a microscopic glass. In this case the formation of a stable whirl is difficult unless the whirl traps an air bubble, which usually sticks to the upper glass on the beam axis ( $c f$. Fig. 3b). When that happens whirl becomes stable and can easily be moved by beam displacement together with the air/gas bubble. The energy stored in this whirl is remarkably high. We proved that the whirl is able to induce the collective movement of micrometric size crystals and displace few micrometer size crystals that are located on its way like a "tornado" (cf. movie SIM2 (ESI ), an air/gas bubble under a microscope looks like a circle with thick black edges).

Frequently, we observed, how the unstable whirl is trying to stabilize its position by vigorously dragging the nearby air/gas bubble even from the distance several times larger than the beam diameter. This means that the liquid even far from the center of the whirl is moved by its dragging force. The movement of liquid is difficult to observe, however when luminescent

insolvable microcrystals are added to the solution they can take part in the fluid flow and demonstrate how the whirl is formed and stopped when the laser beam is blocked. The moving luminescent particles in the whirl have been photographed and are shown in Fig. $3 \mathrm{c}$ on the right side.

\section{B4 Laser-controlled microcrystal growth}

After prolonged exposure, when solution due to solvent evaporation becomes oversaturated the crystallization of $p$-NA is observed. In Fig. 4 and in a video example SIM3 given in the ESI $\neq$ we show, how in a controlled fashion, step by step a single crystal of para-nitroaniline is formed.

First the saturated solution of $p$-NA is formed. When the crystal seed appears crystallization starts. Then with the help of optical trapping that collects $p$-NA from the surrounding one can deliver the new material to the crystal seed by a dedicated movement of the beam with respect to the growing crystal. This opens up a novel technique of crystal growth controlled by light and monitored on screen using a CCD camera coupled with the microscope. The growth could be faster than the conventional one and, which is very important for applications, well controlled allowing growing for example organic nonlinear optical materials and biologically important molecules such as proteins. The process is not new, the pioneering work on laser trap assisted crystallization and the ground of understanding of optical trapping was elaborated and reported by Masuhara's group in several recent papers. ${ }^{37-41,43}$

In Fig. 5 we photographed an interesting sequence of events when a droplet of the $p$-NA condensate grows inside a tubular
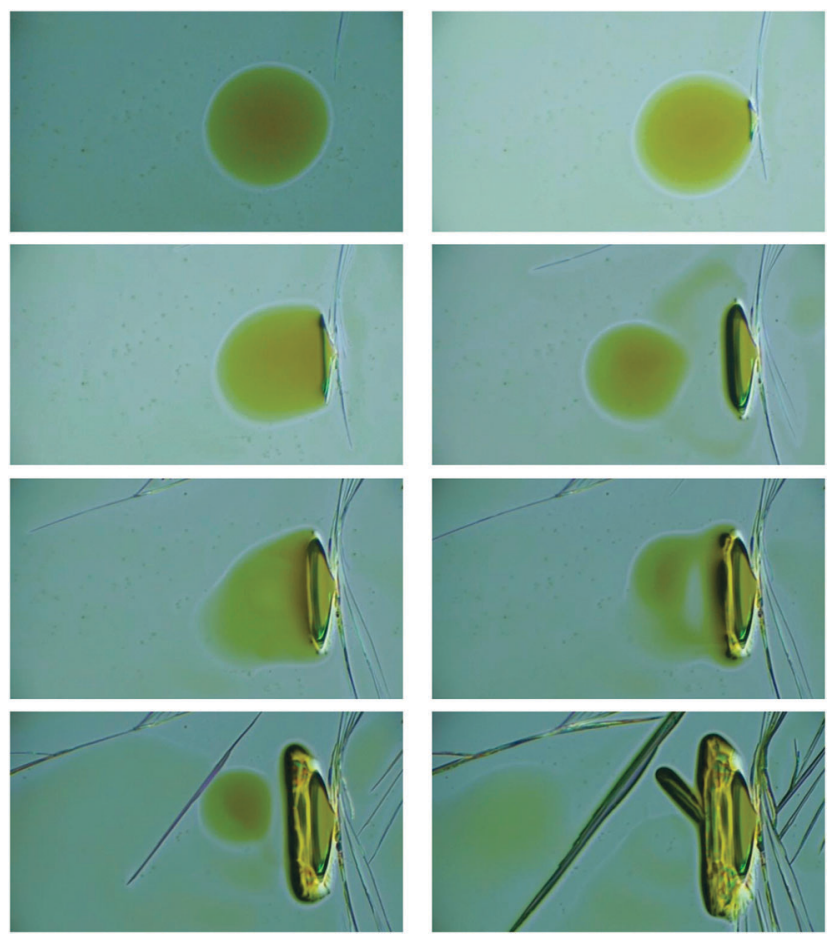

Fig. 4 Sequential $p$-NA crystal growth in 1,4-dioxane solution using the optical trapping mechanism as the supplier of saturated $p$-NA solution in the close vicinity of the crystal growing edge. 

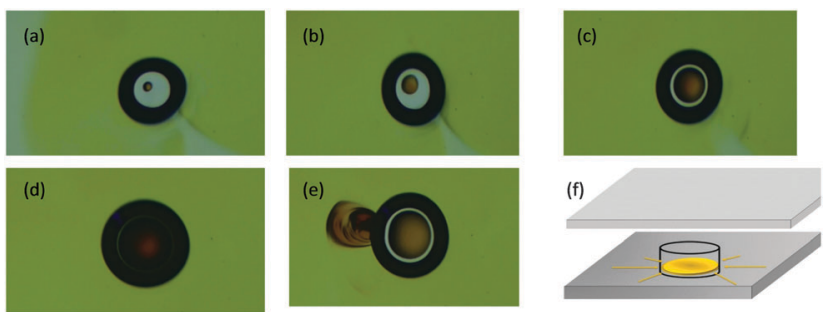

Fig. 5 Experiment performed for a solution of $p$-NA in 1,4-dioxane contained between two glass plates. In the sequence of photographs (a) to (d) droplet formation inside a tubular air tube occurs due to the selective agglomeration of $p$-NA at the beam center. When the size of the droplet approaches that of a tubular air bubble a part of its volume is violently ejected apart (cf. photograph (e)). In figure (f) the scheme of a droplet formed inside a tubular air bubble is shown.

gas/air bubble extending from the bottom to upper glass as shown in Fig. $5 f$. The condensate droplet trapped in the beam center increases its volume due to capillary forces until its size reach the size of the tube, then part of the condensate is violently expelled outside the tube to the surrounding solution ( $c f$. Fig. 5e and video example SIM4, ESI illumination of a laser oversaturates the condensate inside the tube and the material crystallizes exactly at this place. This allows for spatial control over the growth of microscopic size organic crystals.

\section{B5 Discussion of the nature of the trapping mechanism}

The above described phenomena occur in $p$-NA solution close to saturation and for partially absorbed light but under very weak focusing and low cw laser power. All of this excludes the well-known three dimensional optical trapping mechanism reported for dielectric spheres of size larger than several tens of nanometers. ${ }^{44-47}$ The key role, in our understanding of the observed phenomena, is played by the electrostriction mechanism initiating the attraction of streams of liquid by electrostrictive force or gradient light force $\boldsymbol{F}_{\mathrm{g}}(x, y, z)$. Electrostriction is, according to definition, the tendency of materials to become compressed in response to the gradient of an electric field, this field could be the averaged field of electromagnetic waves. In the presence of field $\boldsymbol{E}(x, y, z)$, the molecule develops the dipole moment $\mu=\alpha \boldsymbol{E}$, where $\alpha$ is the molecular polarizability tensor. Weakly focused $\mathrm{cw} \mathrm{TEM}_{00}$ Gaussian beam attracts molecules in the Rayleigh approximation due to Lorentz force. Responsible for the effect is gradient force $\boldsymbol{F}_{\mathrm{g}}(x, y, z)$ :

$$
\boldsymbol{F}_{\mathrm{g}}(x, y, z)=\frac{1}{2} \varepsilon_{\mathrm{m}} \alpha \nabla\left\langle E^{2}\right\rangle
$$

where $\varepsilon_{\mathrm{m}}$ is the electric permittivity of the surrounding medium, $\nabla$ represents a gradient with respect to the spatial coordinates of the beam and the angular brackets denote a time average over an optical field period. This force tries to gather particles in the volume of laser beam focus. Second force is the scattering force $\boldsymbol{F}_{\mathrm{s}}(z)$ which pushes particles axially (along the beam $\boldsymbol{k}$-vector, here the $z$-axis) due to momentum exchange:

$$
\boldsymbol{F}_{\mathrm{s}}(z)=n_{\mathrm{m}} \frac{\langle\boldsymbol{E} \times \boldsymbol{H}\rangle \sigma_{\text {scat }}}{c}
$$

here, $\langle\boldsymbol{E} \times \boldsymbol{H}\rangle=\langle\boldsymbol{S}\rangle$ is the time averaged Poynting vector, $\sigma_{\text {scat }}$ is the light scattering cross section of the particle and $n_{\mathrm{m}}$ is the particle refractive index. Sufficient condition for trapping is that the potential well of the gradient force trap is much larger than the kinetic energy of the Brownian particles ${ }^{45} U$, e.g. $U / k T \geq 10$. The Brownian force, which describes the influence of the Brownian motion, can be calculated as:

$$
\left|\boldsymbol{F}_{\mathrm{b}}\right|=\sqrt{12 \pi \eta a k_{\mathrm{B}} T}
$$

where $\eta$ is the viscosity of the solution $\left(\sim 5 \times 10^{-4} \mathrm{~Pa}\right.$ s), giving a value equal to $\sim 2 \times 10^{-15} \mathrm{~N}$, for a particle with radius $a=$ $50 \mathrm{~nm}$ and $T=300 \mathrm{~K}$ and $\sim 2 \times 10^{-16} \mathrm{~N}$ for a single $p$-NA molecule. Using theory and approximations detailed in the work of Harada et al.,${ }^{47}$ which are adequate to our experiment, and using averaged polarizabilties of $p$-NA $\left(\alpha_{p-\mathrm{NA}}=14.7 \times\right.$ $\left.10^{-24} \mathrm{~cm}^{3}=16.36 \times 10^{-40} \mathrm{Fm}^{2}\right)$ and 1,4-dioxane $\left(\alpha_{\mathrm{d}}=8.6 \times\right.$ $\left.10^{-24} \mathrm{~cm}^{3}=9.569 \times 10^{-40} \mathrm{Fm}^{2}\right)^{48}$ we prove that the gradient force is unable to trap even $100 \mathrm{~nm} p$-NA particles for which the maximum gradient force at a distance equal to half of the beam waist (here $50 \mu \mathrm{m}$ ) amounts merely to $F_{\mathrm{g}, \max }=1.2 \times 10^{-11} \mathrm{pN}$. The gradient force is proportional to particle polarizability given for a spherical particle as:

$$
\alpha=4 \pi a^{3} \frac{\left(\frac{n_{\mathrm{c}}}{n_{\mathrm{d}}}\right)^{2}-1}{\left(\frac{n_{\mathrm{c}}}{n_{\mathrm{d}}}\right)^{2}+2}
$$

where $n_{\mathrm{c}}$ is the refractive index of the condensate (here $n_{\mathrm{c}} \approx n_{p \text {-NA }}=$ 1.634 and $n_{\mathrm{d}} \approx 1.404$ stays for the solvent) and $a$ is the spherical particle radius. For weakly focused light one can rather expect attraction of the liquid toward the beam center occurring practically in two dimensions $(x, y)$ because along the $z$-axis electrostrictive forces are much weaker. So the molecules which enter the beam area can move up or down but have difficulty in escaping from the center of the beam.

Generally, the molecule accumulation process is slow because it occurs only within an area of radius $r_{0}$ from the beam center for which attractive forces are sufficiently large. In the described case, the dragged particles move preferentially in one direction causing the flow of fluid along the light $\boldsymbol{k}$-vector and drag the fluid from a large volume of the surrounding beam area. For constant illumination solution in the central region of the beam will fast be enriched with $p$-NA molecules which will result in a gradual increase of fluid density (the density of $p$-NA is $1.333 \mathrm{~g} \mathrm{~cm}^{-3}$ while the density of 1,4-dioxane is $0.995 \mathrm{~g} \mathrm{~cm}^{-3}$ ). At the same time the surface tension of the highly concentrated $p$-NA solution will increase as surface tension for $p$-NA is $\sigma_{p \text {-NA }}=60.3$ dynes per $\mathrm{cm}$ and that of 1,4-dioxane is $\sigma_{\text {dioxane }}=31.2$ dynes per $\mathrm{cm}$. When a high enough concentration of $p$-NA molecules is established a light induced phase separation takes place resulting in a convexshaped liquid dense droplet formation on a glass support, laterally reaching the millimeter size. This is possible due to van der Waals molecular interaction and possibly weak H-bonding forming various size $p$-NA agglomerates of high total polarizability 
(van der Waals potential scales as UvW $\sim l^{-6}$, where $l$ is the average separation between $p$-NA molecules in the condensate). In the enriched $p$-NA liquid droplet having its well defined phase volume due to increase in light absorption an intense production of heat along the $z$-axis will start. This effect will accelerate the mass convection toward the surface area. The streams of the hot condensate cool down at the surface, mostly due to the evaporation heat of 1,4-dioxane and return by sides of the droplet to its bottom and are forced to move again up mostly within the volume of the condensate which gradually increases. The formed liquid droplet is stably observed until the trapping laser is switched on. This type of surface tension convection, known as Maragoni convection, was discussed thoroughly in ref. 49. The surface tension convection enhanced with buoyant force makes an upward fluid motion, i.e. a whirl. The fluid velocity distribution inside the whirl could be modeled using an appropriate solution of the Navier-Stokes equation. The flux velocity of liquid increases with time due to temperature gradient increase caused by the $p$-NA fluid enrichment process and decreases due to the augmentation of kinematic viscosity $\eta$ when the fluid becomes more densely packed with $p$-NA particles. Droplet formation in solution covered by glass is more complex as no free solvent evaporation takes place. The whirl in this case is formed preferably by trapping a gas/air bubble at its top to facilitate the smooth flow of streams of liquid. Sometimes when the whirl rotation speed is too high to accommodate the liquid flowing inside, the conditions of whirl's stability are not fulfilled and, as a consequence, a part of its mass is ejected vigorously outside the droplet border to the surrounding solution (see video SIM4, ESI ). The mass ejection phenomenon has been reported for the femtosecond optical trapping of polystyrene nanoparticles in water by Chiang et al. ${ }^{50}$ The direction of mass removal from the trap in the experiment described in ref. 50 was correlated with the direction of linear light polarization being perpendicular to the plane of laser beam polarization. In our case ( $c f$. Fig. 5(e)) the mechanism is obviously different and its correlation with light polarization needs further studies.

\section{Conclusions}

In conclusion, the laser-light induced phenomena described in this article in liquids containing dissolved molecules are quite general ones and their proper understanding requires systematic studies. The laser-induced coupling between optical lateral attraction of the molecules due to the electrostrictive mechanism and large liquidlike cluster formation allows for liquid-liquid phase separation. The increase in local molecular concentration enhanced by convection loop formation followed by the flow of solution along the beam center (the whirl) is a novel unexplored tool enabling molecular sorting. This phenomenon can easily lead to a large increase of material concentration at the beam position and controlled nucleation and crystal growth under a microscope.

\section{Acknowledgements}

This work was financially supported by the Polish National Science Centre (Dec-2011/03/B/ST5/01021).

\section{References}

1 A. Ashkin, Phys. Rev. Lett., 1970, 24, 156.

2 A. Ashkin, Proc. Natl. Acad. Sci. U. S. A., 1997, 94, 4853.

3 M. J. Lang and S. M. Block, Am. J. Phys., 2003, 71, 201.

4 P. J. Allen, Am. J. Phys., 1966, 34, 1185.

5 M. E. J. Friese, J. Enger, H. Rubinsztein-Dunlop and N. R. Heckenberg, Phys. Rev. A: At., Mol., Opt. Phys., 1996, 54, 1593.

6 M. Padgett and R. Bowman, Nat. Photonics, 2011, 5, 343.

7 D. G. Grier, Nature, 2003, 424, 810.

8 S. Parkin, G. Knöner, W. Singer, T. A. Nieminen, N. R. Heckenberg and H. Rubinsztein-Dunlop, Methods Cell Biol., 2007, 82, 525.

9 D. Hakobyan and E. Brasselet, Nat. Photonics, 2014, 8, 610.

10 H. Misawa, K. Sasaki, M. Koshioka, N. Kitamura and H. Masuhara, Appl. Phys. Lett., 1992, 60, 310.

11 P. Galajda and P. Ormos, Appl. Phys. Lett., 2001, 78, 249.

12 S. Juodkazis, S. Matsuo, N. Murazawa, I. Hasegawa and H. Misawa, Appl. Phys. Lett., 2003, 82, 4657.

13 C. Manzo, D. Paparo, L. Marrucci and I. Janossy, Phys. Rev. E: Stat., Nonlinear, Soft Matter Phys., 2006, 73, 051707.

14 E. Brasselet and L. J. Dube, Phys. Rev. E: Stat., Nonlinear, Soft Matter Phys., 2006, 73, 021704.

15 M. P. MacDonald, G. C. Spalding and K. Dholakia, Nature, 2003, 426, 421.

16 A. Bishop, T. Nieminen, N. Heckenberg and H. RubinszteinDunlop, Phys. Rev. Lett., 2004, 92, 198104.

17 C. Bustamante, Z. Bryant and S. Smith, Nature, 2003, 421, 423.

18 H. Jensenius and G. Zocchi, Phys. Rev. Lett., 1997, 79, 5030.

19 N. Murazawa, S. Juodkazis, S. Matsuo and H. Misawa, Small, 2005, 1, 656 .

20 H. Misawa and S. Juodkazis, Prog. Polym. Sci., 1999, 24, 665.

21 Y. Nabetani, H. Yoshikawa, A. C. Grimsdale, K. Mullen and H. Masuhara, Langmuir, 2007, 23, 6737.

22 Y. Nabetani, H. Yoshikawa, A. Grimsdale, K. Mullen and H. Masuhara, Jpn. J. Appl. Phys., 2007, 46, 449.

23 W. Singer, T. A. Nieminen, N. R. Heckenberg and H. Rubinsztein-Dunlop, Phys. Rev. E: Stat., Nonlinear, Soft Matter Phys., 2007, 75, 011916.

24 S. Ito, Y. Tanaka, H. Yoshikawa, Y. Ishibashi, H. Miyasaka and H. Masuhara, J. Am. Chem. Soc., 2011, 133, 14472.

25 J. Hofkens, J. Hotta, K. Sasaki, H. Masuhara and K. Iwai, Langmuir, 1997, 13, 414.

26 Y. Tanaka, H. Yoshikawa and H. Masuhara, J. Phys. Chem. B, 2006, 110, 17906.

27 Y. Tsuboi, T. Shoji and N. Kitramura, J. Phys. Chem. C, 2010, 114, 5589.

28 J. Hotta, K. Sasaki and H. Masuhara, J. Am. Chem. Soc., 1996, 118, 11968.

29 L. Jauffred, A. C. Richardson and L. B. Oddershede, Nano Lett., 2008, 8, 3376.

30 S. Ito, H. Yoshikawa and H. Masuhara, Appl. Phys. Lett., 2001, 78, 2566.

31 C. Hosokawa, H. Yoshikawa and H. Masuhara, Phys. Rev. E: Stat., Nonlinear, Soft Matter Phys., 2005, 72, 021408. 
32 Y. Tanaka, H. Yoshikawa, T. Itoh and M. Ishikawa, Opt. Express, 2009, 17, 18760.

33 Y. Zhang and C. Gu, Phys. Rev. B: Condens. Matter Mater. Phys., 2006, 73, 165405.

34 K. Yuyama, T. Sugiyama and H. Masuhara, J. Phys. Chem. Lett., 2010, 1, 1321.

35 A. Usman, T. Uwada and H. Masuhara, J. Phys. Chem. C, 2001, 115, 11906.

36 A. Kotnala and R. Gordon, Nano Lett., 2014, 14, 853.

37 S. Ito, Y. Tanaka, H. Yoshikawa, Y. Ishibashi, H. Miyasaka and H. Masuhara, J. Am. Chem. Soc., 2011, 133, 14472.

38 T. Sugiyama, K. Yuyama and H. Masuhara, Acc. Chem. Res., 2012, 45, 1946.

39 J. Tu, K. Yuyama, H. Masuhara and T. Sugiyama, Cryst. Growth Des., 2013, 14, 15.

40 K. Yuyama, T. Sugiyama and H. Masuhara, J. Phys. Chem. Lett., 2013, 4, 2436.
41 T. Sugiyama and H. Masuhara, Chem. - Asian J., 2011, 6, 2878.

42 T. A. Nieminen, N. Preez-Wilkinson, A. B. Stilgoe, V. L. Y. Loke, A. A. M. Bui and H. Rubinsztein-Dunlop, J. Quant. Spectrosc. Radiat. Transfer, 2014, 146, 59.

43 K. Yuyama, T. Rungsimanon, T. Sugiyama and H. Masuhara, J. Phys. Chem. C, 2012, 116, 6809.

44 A. Ashkin and J. M. Dziedzic, Appl. Phys. Lett., 1971, 19, 283. 45 A. Ashkin, J. M. Dziedzic, J. E. Bjorkholm and S. Chu, Opt. Lett., 1986, 11, 288.

46 M. J. Lang and S. M. Block, Am. J. Phys., 2003, 71, 201.

47 Y. Harada and T. Asakura, Opt. Commun., 1996, 124, 529.

48 L. Jensen and P. T. van Duijnen, J. Chem. Phys., 2005, 123, 074307.

49 O. A. Luochev, S. Juodkazis, N. Murazawa, S. Wada and H. Misawa, Opt. Express, 2008, 6, 5673.

50 W. Chiang, A. Usman and H. Masuhara, J. Phys. Chem. C, 2013, 117, 19182. 